Supporting information for

\title{
Block Copolymer Derived Vertically Coupled Plasmonic Arrays for Surface Enhanced Raman Spectroscopy
}

Goekalp Engin Akinoglu ${ }^{1,2,3,}$, Sajjad Husain Mir $^{1,2}$, Riley Gatensby ${ }^{1,2}$, Gaulthier Rydzek ${ }^{1,2}$, Parvaneh Mokarian-Tabari ${ }^{1,2, *}$

${ }^{1}$ Advanced Materials and BioEngineering Research Centre (AMBER), Trinity College Dublin, The University of Dublin, Dublin 2, Ireland

${ }^{2}$ The School of Chemistry, Trinity College Dublin, The University of Dublin, Dublin 2, Ireland

${ }^{3}$ Freie Universität Berlin, Department of Physics, 14195 Berlin, Germany

E-mail: akinoglg@tcd.ie

E-mail: parvaneh.mokarian@tcd.ie
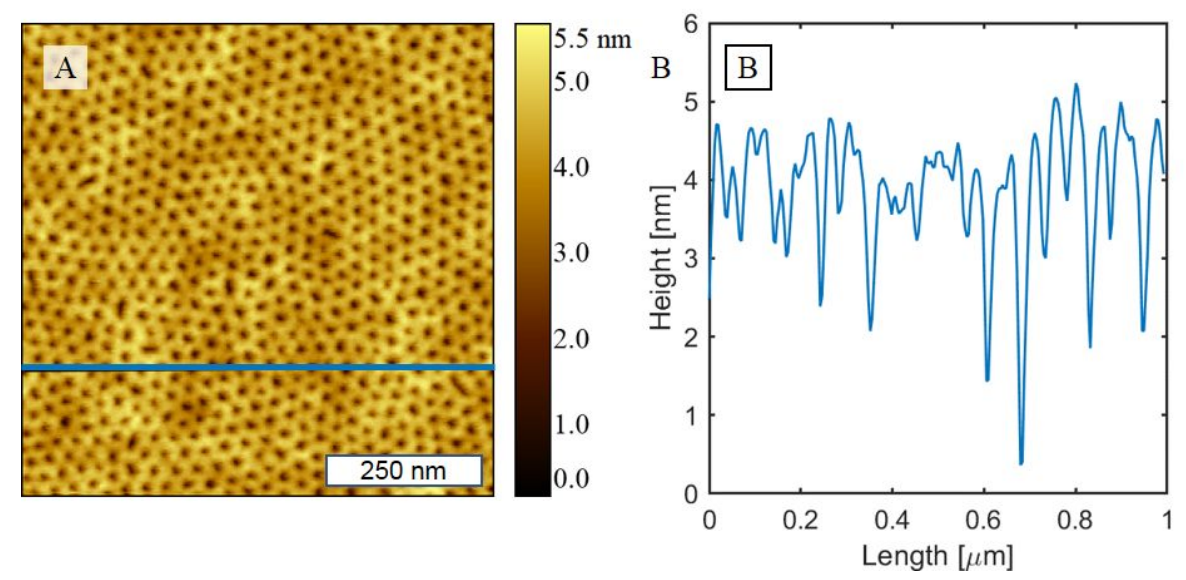

Figure S1. PS-b-PEO after phase separation. (A) AFM picture. (B) AFM profile line.
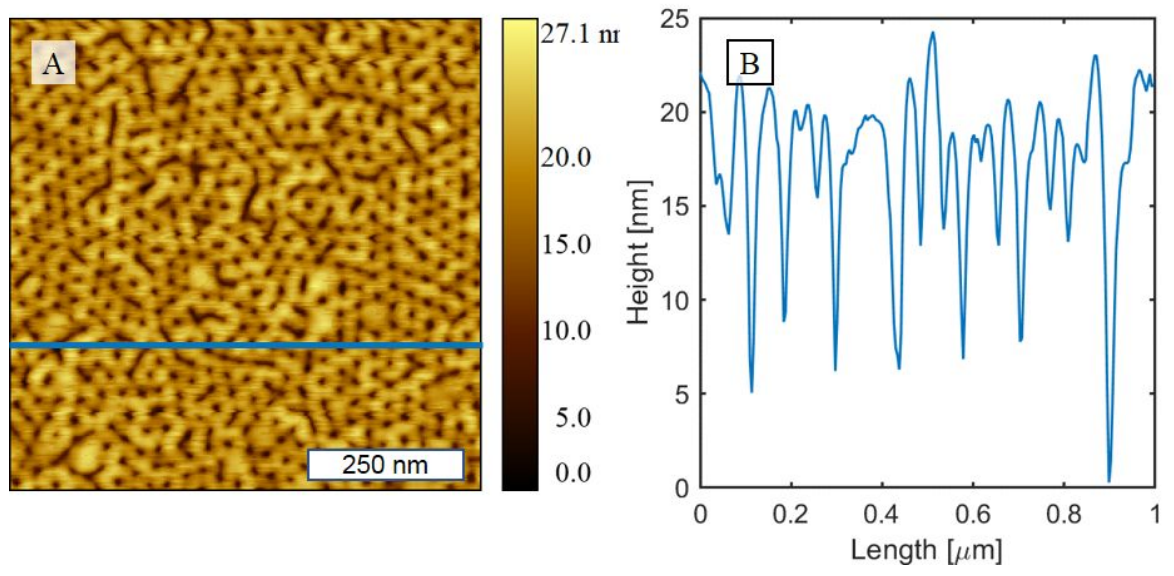

Figure S2. PS-b-PEO after swelling. (A) AFM picture. (B) AFM profile line. 

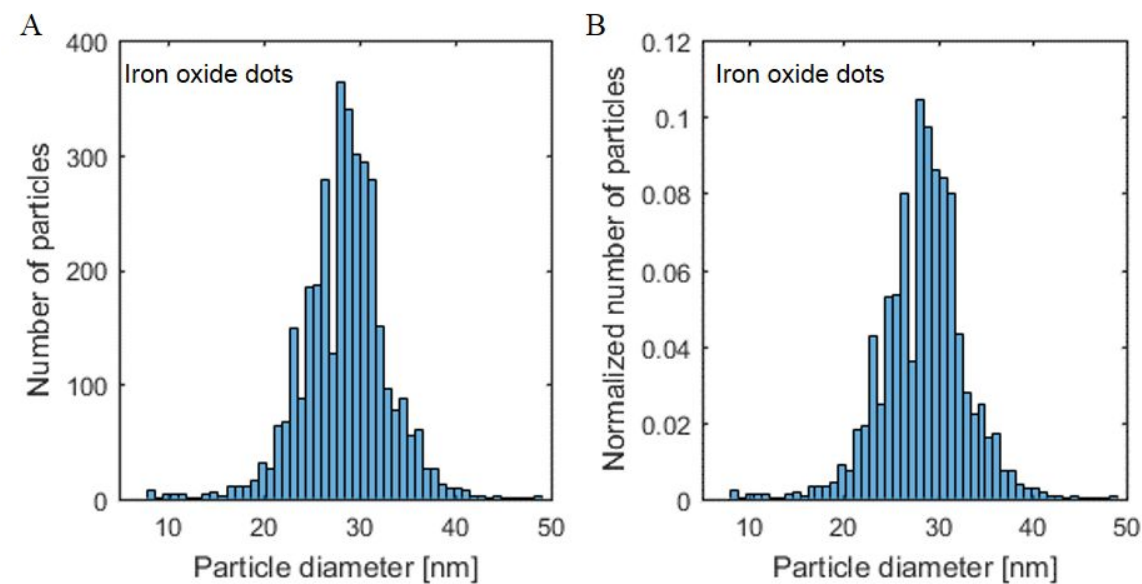

Figure S3. Particle size distribution of the iron oxide particles: (A) absolute and (B) relative
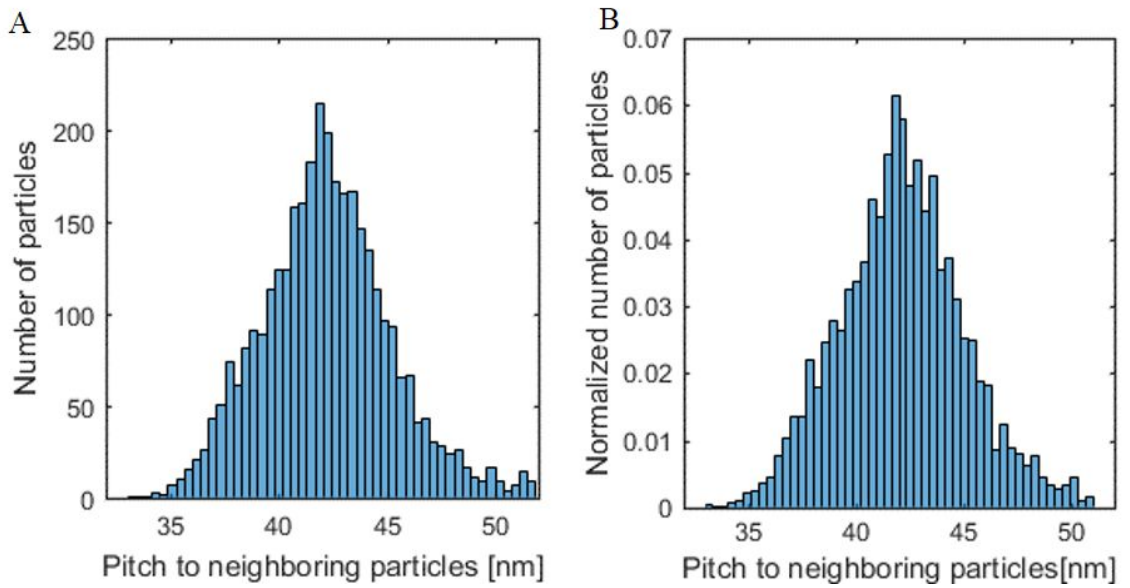

Figure S4. Iron oxide nanoparticle pitch: (A) absolute and (B) relative.
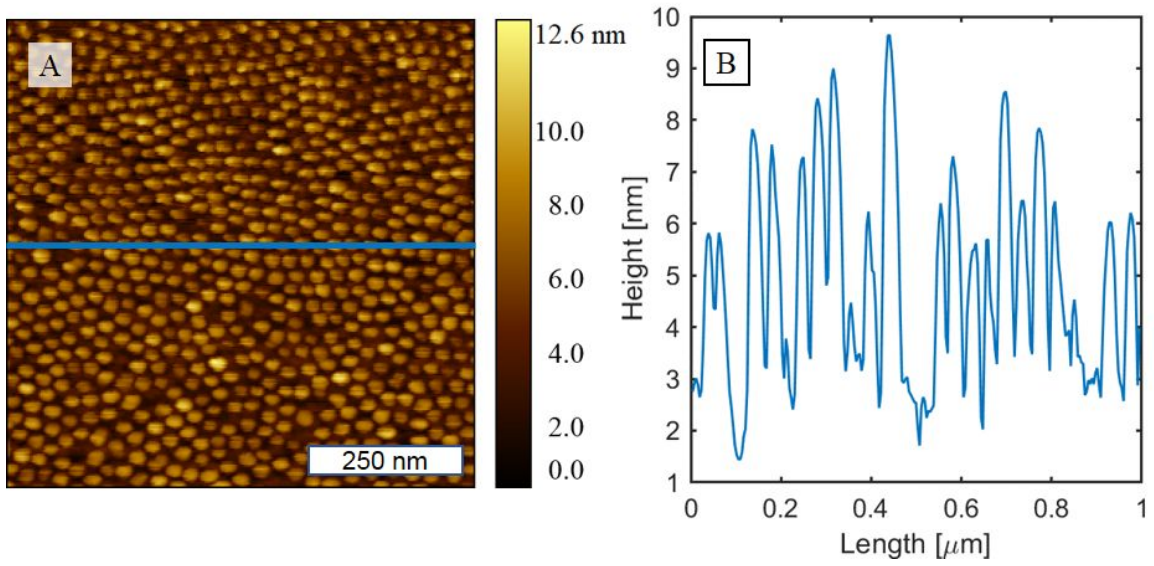

Figure S5. Iron oxide hard mask (A) AFM picture. (B) AFM profile line. The AFM profile underestimates the pillar height because the AFM tip apex is too large to fully penetrate the etched trenches due to its size. 

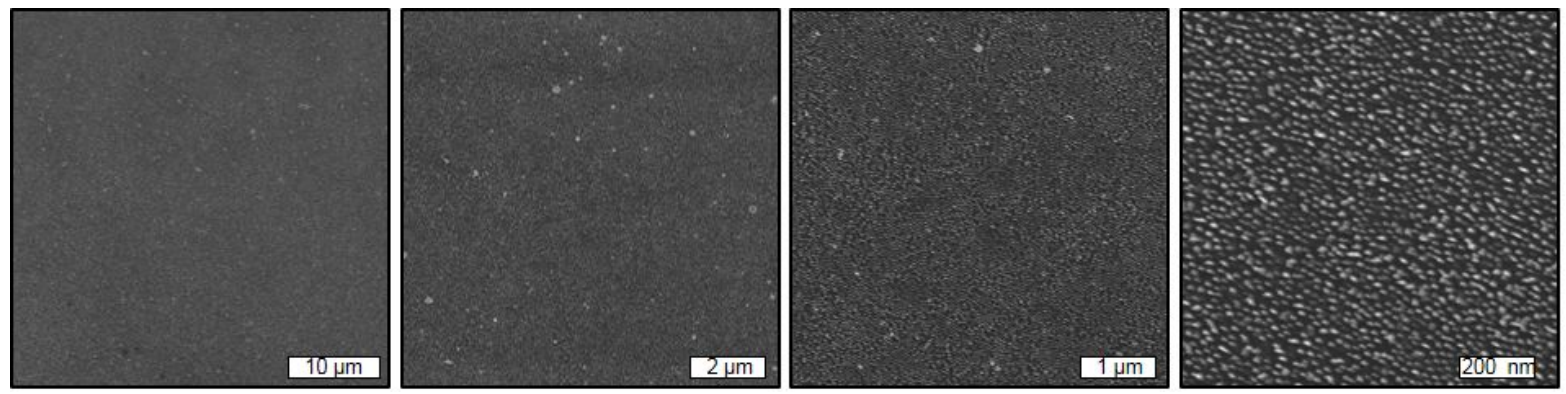

Figure S6. SEM top view with different magnifications of nanopillars before iron oxide removal
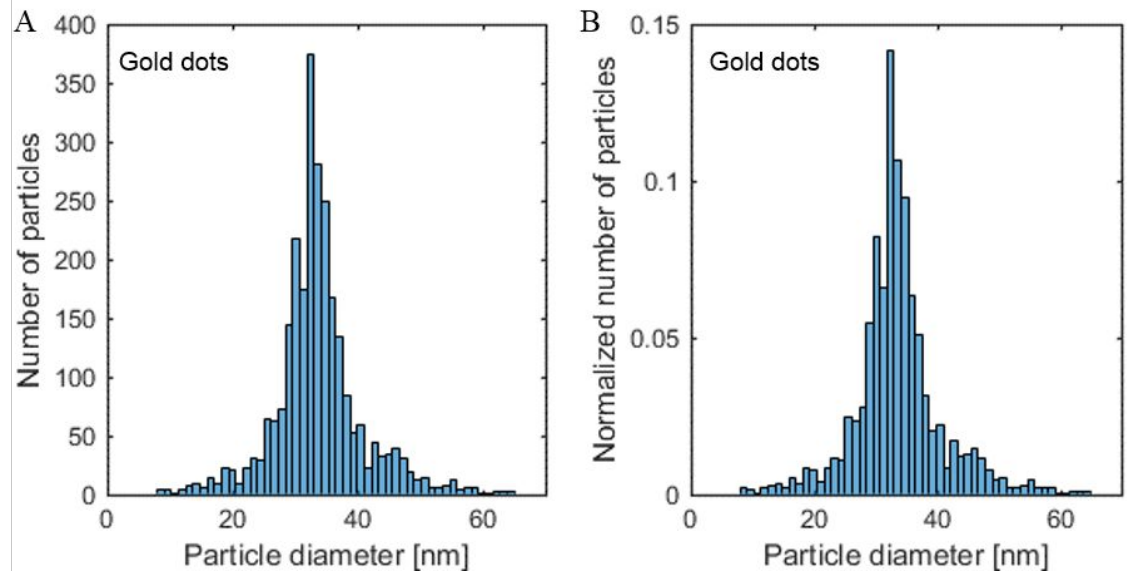

Figure S7. Particle size distribution of the gold dots on top of the nanopillars: (A) absolute and $(\mathrm{B})$ relative.
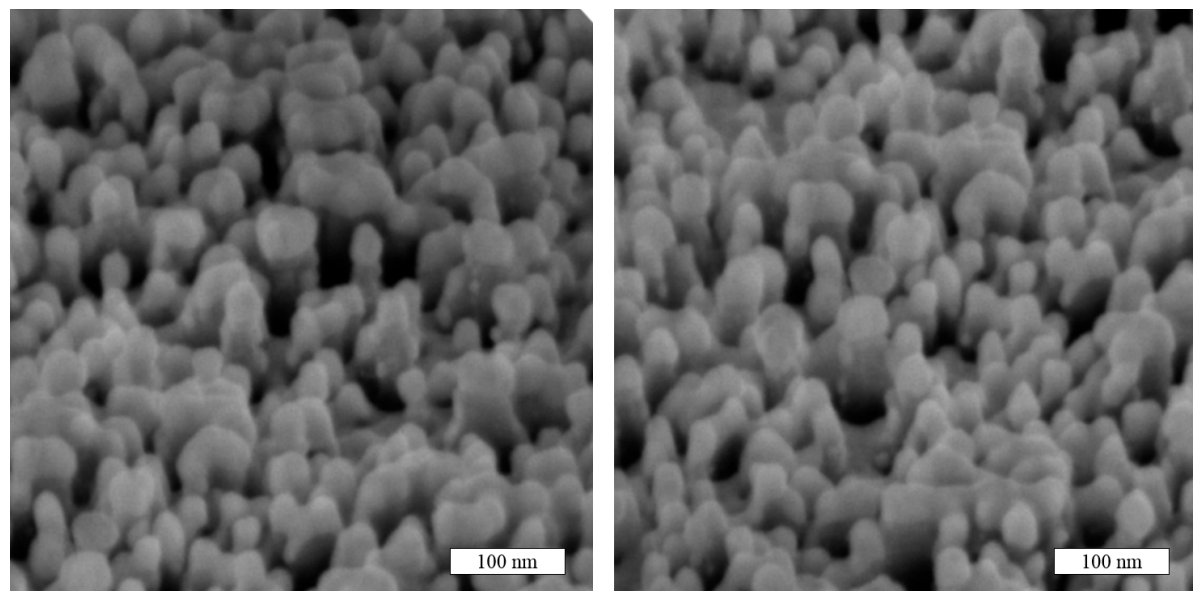

Figure S8. Tilted SEM pictures of VCPAs at a defect zone. 


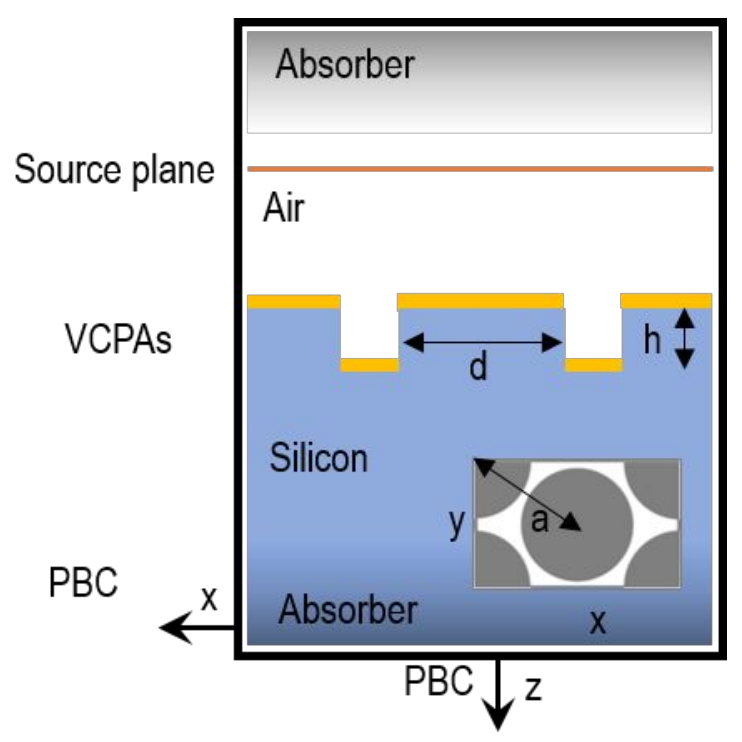

Figure S9. Cross section of the FDTD simulation cell. The pillar diameter $d$, the pillar height $h$ and the pitch $a$ define the dimensions of the VCPAs. A periodic boundary conditions (PBC) is used and is terminated along the z-axis by an artificial absorber layer. 